\title{
Three-Dimensional Interconnected Microporous Carbon Network Derived from Aniline Formaldehyde Resin/Sodium Polyacrylate Interpenetrating Polymer Networks (AF/PAAS IPNs) with Controllable Porosity for Supercapacitors
}

\author{
Yan-Dong Ma ${ }^{\mathrm{a}}$, Xi-Wen Chen ${ }^{\mathrm{a}}$, and Ling-Bin Kong ${ }^{* a, b}$
}

\section{Experimental Section}

\section{Materials}

Aniline (analytical grade), formaldehyde (37 wt.\%), sodium hydrate $(\mathrm{NaOH})$, analytically pure potassium hydrate $(\mathrm{KOH})$, hydrochloric acid $(\mathrm{HCl})$ and ethanol were all purchased from Sigma-Aldrich. Sodium polyacrylate (PAAS, the main ingredient of super absorbent polymer) was supplied by ANJUHUANBAO Co. Ltd., China. The aniline can be used after distillation at $115^{\circ} \mathrm{C}$ under reduced pressure, and other chemical reagents were used as received without further purification procedures. Distilled deionized water was employed for all the experiments.

\section{Synthesis of AF/PAAS IPNs}

In a typical experimental procedure, $2 \mathrm{~g}$ of aniline was dissolved in $100 \mathrm{ml}$ of deionized water with magnetic stirring in a glass stopper flask and the $\mathrm{pH}$ was adjusted to $8-9$ with $1 \mathrm{~mol} \mathrm{~L}^{-1}$ aqueous $\mathrm{NaOH}$. Then, $1.63 \mathrm{ml}$ of an aqueous formaldehyde solution (mass fraction of $37 \%$ ) was carefully added. At this point it can be seen that the solution turned from colorless and transparent to a white emulsion. Gradually add PAAS and stir for a while until the solution turns into a white gel. The AF prepolymer/PAAS gel was transferred to a $100 \mathrm{ml}$ Teflon autoclave vessel and hydrothermally treated in an oven at $100{ }^{\circ} \mathrm{C}$ for 6 hours. After naturally cooling, it was dried in an 
oven at $60{ }^{\circ} \mathrm{C}$ for 24 hours to obtain AF/PAAS IPNs. The specific experimental process is shown in scheme 1. We also synthesized a blend of AF and PAAS and a pure AF resin for comparison under the same conditions (scheme 1). To further explore the effect of the content of aniline formaldehyde resin on the pore structure and electrochemical properties of the resulting carbon material, we obtain a series of IPNs samples by controlling the content of aniline $(2 \mathrm{~g}, 2.5 \mathrm{~g}, 3 \mathrm{~g}$, $3.5 \mathrm{~g}$, and $4 \mathrm{~g}$ ) and ensuring other conditions (including the molar ratio of aniline and formaldehyde is 1:1) are constant.

\section{Preparation of MC-IPNx}

The obtained IPNs was ground to a fine powder. Then, porous carbon was produced by carbonizing the IPNs powder under an $\mathrm{N}_{2}$ atmosphere for 2 hours. The final temperature and heating rate of the furnace were $950{ }^{\circ} \mathrm{C}$ and $2{ }^{\circ} \mathrm{C} / \mathrm{min}$, respectively. The product is expressed as MC-IPNx $(\mathrm{x}=1 \sim 5$ representing the different aniline contents from $2 \mathrm{~g} \sim 4 \mathrm{~g}$ mentioned in the synthetic AF/PAAS IPNs step, respectively. For comparison, a blend of AF and PAAS and a pure $\mathrm{AF}$ resin were carbonized, respectively, and the obtained carbons were named C-AFB and C-AF.

\section{Structural characterization}

Fourier transform infrared (FTIR) spectra were measured using a Bruker Equinox 55 FTIR spectrophotometer. Samples were synthesized by casting pellets from sample/KBr mixtures. Power X-ray diffraction (XRD) patterns were measured on the Bruker Focus D8 diffractometer with $\mathrm{Cu} \mathrm{K} \alpha$ radiation $(40 \mathrm{kV}, \lambda=0.15418 \mathrm{~nm})$ between $10^{\circ}$ and $80^{\circ}$. Raman spectras were obtained by a Renishaw invia spectrometer with a $520 \mathrm{~nm}$ Ar-ion laser. The morphologies and structures of the samples were obtained using scanning electron microscopy (SEM) and transmission electron microscopy (TEM, $200 \mathrm{kV}$ ). Pore structure was researched using the JWGB surface area and 
porosity analyzer. Specific surface areas were analyzed by Brunauer-Emmett-Teller (BET) theory. The pore size distribution (PSD) was calculated according to the Horvath-Kawazoe (H-K) model (for microporous) and Barret-Joyner-Halenda (BJH) model (for mesopores and macropores). The total pore volumes were calculated from the amount adsorbed at a relative pressure $P / P_{0}$ of 0.99 .

\section{Electrochemical characterization}

MC-IPNs electrode was obtained by mixing electroactive material powder $(80 \mathrm{wt} \%)$, conductive graphite $(7.5 \mathrm{wt} \%)$, acetylene black (7.5 wt \%) and polytetrafluorethylene (PTFE, $5 \mathrm{wt} \%$ dispersed in water) in ethanol. The mixture was pressed into a foamed nickel current collector (surface area: $1 \mathrm{~cm} * 1 \mathrm{~cm}$ ). The mass of the electroactive material powder in each electrode was about $4 \mathrm{mg}$. The foamed nickel was pressed at $10 \mathrm{MPa}$ after which was dried in a vacuum oven at $60{ }^{\circ} \mathrm{C}$ for 12 hours. The electrochemical properties of the EDLC were measured using an electrochemical workstation (CHI660E, Shanghai, China): cyclic voltammetry (CV) was obtained at different scan rates; galvanostatic charge-discharge (GCD) curves were measured at various current densities. The specific capacitance $\left(\mathrm{C}_{\mathrm{g}}\right)$ is calculated according to the equation $\mathrm{C}_{\mathrm{g}}=\mathrm{I} \times \Delta \mathrm{t}$ $/(\mathrm{m} \times \Delta \mathrm{V})$, where $\mathrm{C}_{\mathrm{g}}\left(\mathrm{F} \mathrm{g}^{-1}\right), \mathrm{m}(\mathrm{g}), \mathrm{I}(\mathrm{A}), \Delta \mathrm{t}(\mathrm{s})$ and $\Delta \mathrm{V}(\mathrm{V})$ represent specific capacitance, working electrode mass, constant current density, discharge time and discharge potential respectively. The electrochemical impedance spectroscopy (EIS) measurement was performed at an open circuit potential in the frequency range of $10^{5}$ to $10^{-2} \mathrm{~Hz}$ with an AC perturbation of $5 \mathrm{mV}$. 
(a)

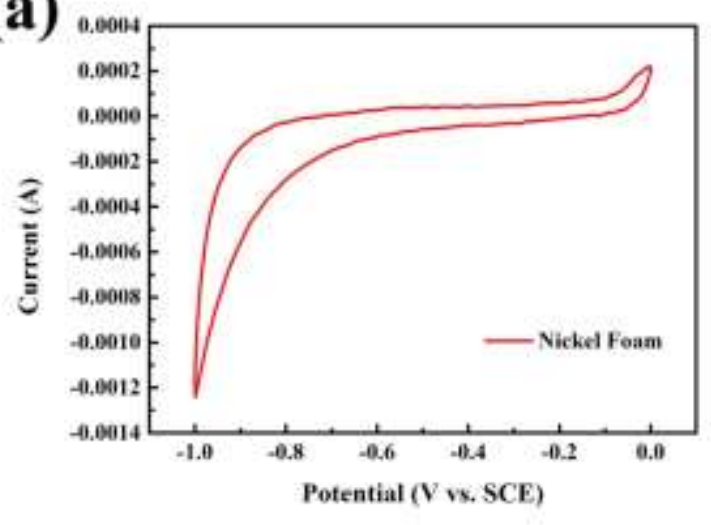

(b)

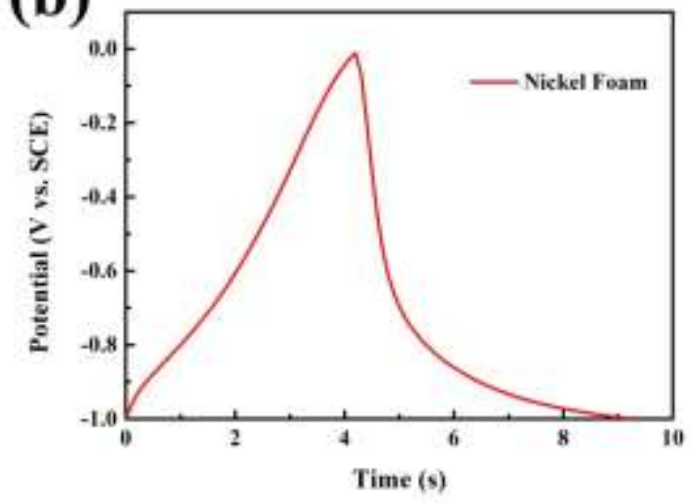

Figure S1. Electrochemical performances of Nickel Foam: (a) CV curves at $5 \mathrm{mV} \mathrm{s}^{-1}$, (b) GCD curves at $0.5 \mathrm{~A} \mathrm{~g}^{-1}$.
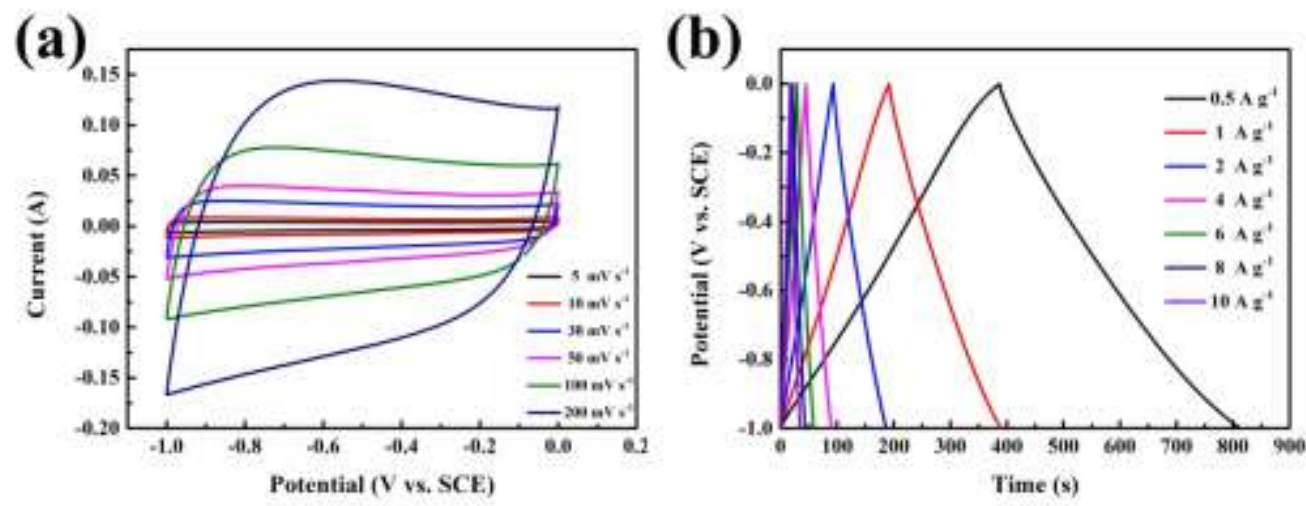

Figure S2. Electrochemical performances of C-IPN: (a) CV curves, (b) GCD curves.

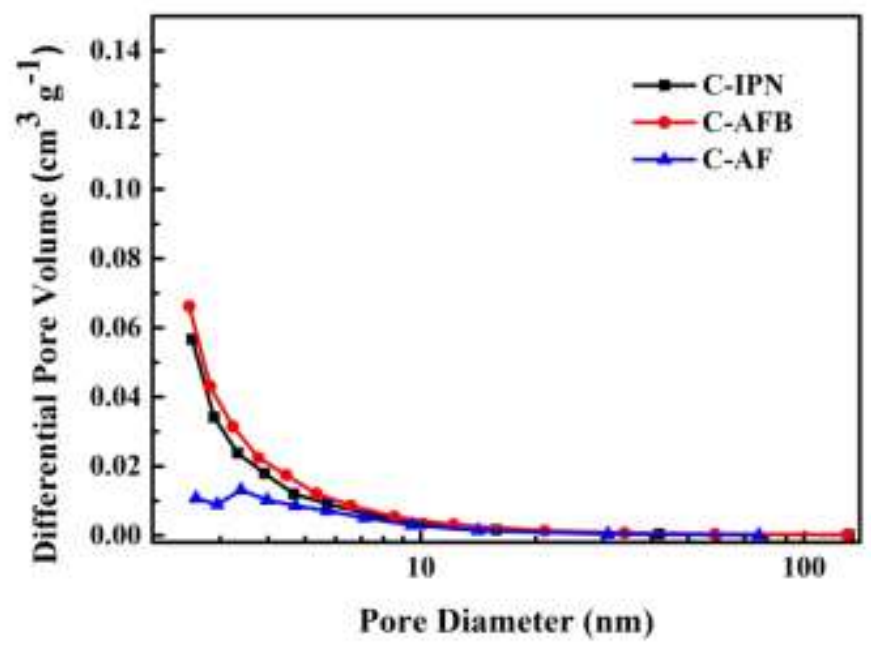

Figure S3. The pore size distributions $(2.3-110 \mathrm{~nm})$ of the samples synthesized under 
different conditions.

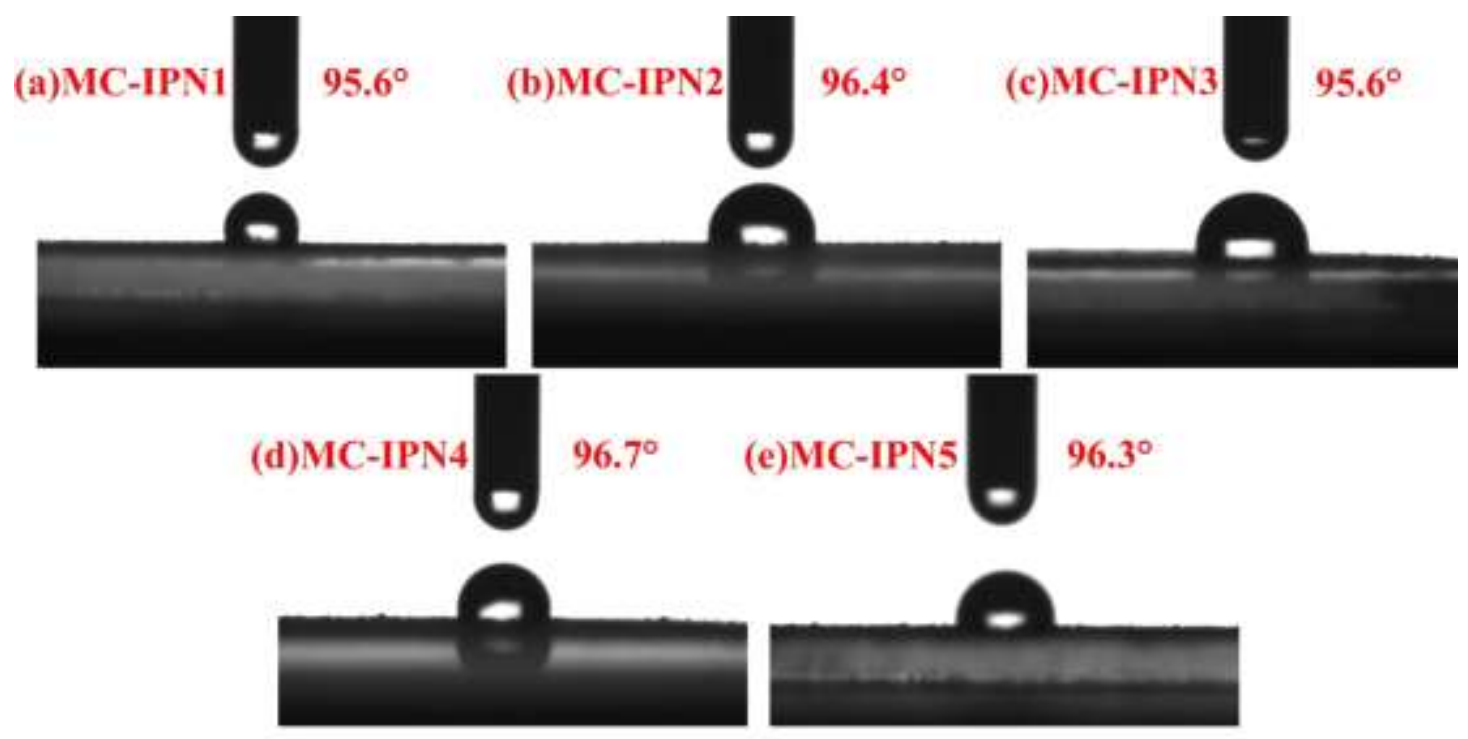

Figure S4. The water contact angles of MC-IPNx.
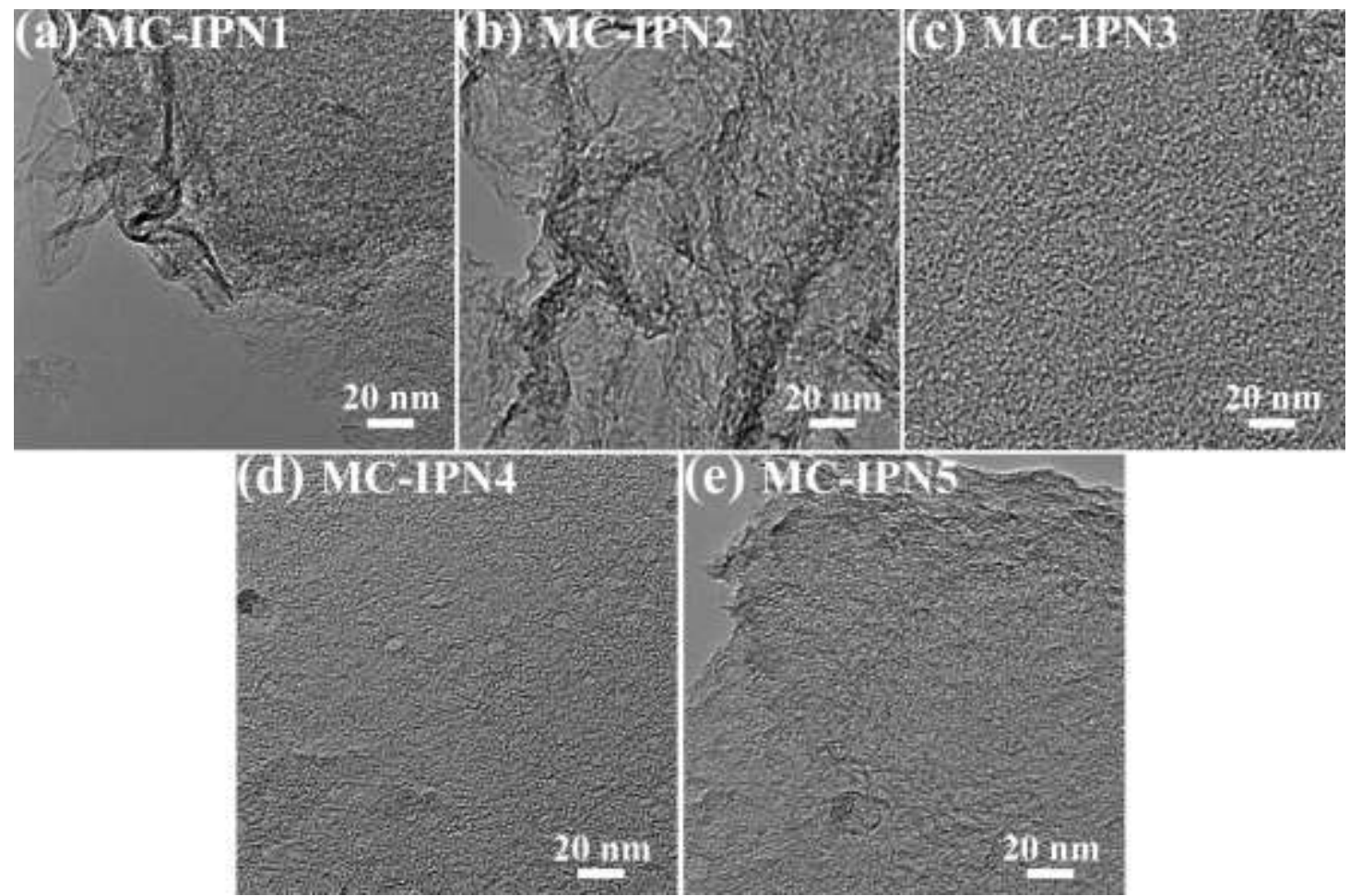

Figure S5. The TEM images of (a) MC-IPN1, (b) MC-IPN2, (c) MC-IPN3, (d) MC-IPN4, and (e) MC-IPN5. 